\title{
L-Topological Spaces Based on Residuated Lattices
}

\author{
Zhudeng Wang ${ }^{1}$, Xuejun Liu ${ }^{2}$ \\ ${ }^{1}$ Department of Mathematics, Yancheng Teachers University, Yancheng, China \\ ${ }^{2}$ School of Computer and Information Technology, Zhejiang Wanli University, Ningbo, China \\ Email: zhudengwang2004@yahoo.com.cn, Im88134005@126.com
}

Received September 14, 2011; revised October 10, 2011; accepted October 20, 2011

\begin{abstract}
In this paper, we introduce the notion of $L$-topological spaces based on a complete bounded integral residuated lattice and discuss some properties of interior and left (right) closure operators.
\end{abstract}

Keywords: Residuated Lattice; L-Topological Space; Interior Operator; Left (Right) Closure Operator

\section{Introduction}

Residuation is a fundamental concept of ordered structures and the residuated lattices, obtained by adding a residuated monoid operation to lattices, have been applied in several branches of mathematics, including $L$-groups, ideal lattices of rings and multivalued logic. Commutative residuated lattices have been studied by Krull, Dilworth and Ward. These structures were generalized to the non-commutative situation by Blount and Tsinakis [1].

Definition 1.1. [1-4]. A residuated lattice is an algebra $L=(L, \wedge, \vee, \cdot, \rightarrow, \mapsto, 0,1)$ of type $(2,2,2,2,2,0,0)$ satisfying the following conditions:

(L1) $(L, \wedge, \vee)$ is a lattice,

(L2) $(L, \cdot, 1)$ is a monoid, i.e., - is associative and $x \cdot 1=1 \cdot x=x$ for any $x \in L$,

(L3) $x \cdot y \leq z$ if and only if $x \leq y \rightarrow z$ if and only if $y \leq x \mapsto z$ for any $x, y, z \in L$.

Generally speaking, 1 is not the top element of $L$. A residuated lattice with a constant 0 is called a pointed residuated lattice or full Lambek algebra ( $F L$-algebra, for short). If $x \leq 1$ for all $x \in L$, then $L$ is called integral residuated lattice. An $F L$-algebra $L$ which satisfies the condition $0 \leq x \leq 1$ for all $x \in L$ is called $F L_{w}$-algebra or bounded integral residuated lattice (see [2]). Clearly, if $L$ is an $F L_{w}$-algebra, then $(L, \wedge, \vee, 0,1)$ is a bounded lattice.

A bounded integral residuated lattice is called commutative (see [5]) if the operation - is commutative. We adopt the usual convention of representing the monoid operation by juxtaposition, writing $a b$ for $a \cdot b$.

The following theorem collects some properties of bounded integral residuated lattices (see [1-4,6].

Theorem 1.1. Let $L$ be a bounded integral residuated lattice. Then the following properties hold.

1) $x \rightarrow x=x \mapsto x=1,1 \rightarrow x=1 \mapsto x=x$.
2) $x \rightarrow(y \mapsto z)=y \mapsto(x \rightarrow z)$.

3) $x(x \mapsto y) \leq x \wedge y,(x \rightarrow y) x \leq x \wedge y, x \leq y \rightarrow x y$, $y \leq x \mapsto x y$.

4) $(x \mapsto y)(y \mapsto z) \leq x \mapsto z$, $(y \rightarrow z)(x \rightarrow y) \leq x \rightarrow z$.

5) If $x \leq y$, then $x z \leq y z, z x \leq z y, x \rightarrow z \geq y \rightarrow z$, $x \mapsto z \geq y \mapsto z, z \rightarrow x \leq z \rightarrow y$ and $z \mapsto x \leq z \mapsto y$.

6) $x \leq y$ if and only if $x \rightarrow y=1$ if and only if $x \mapsto y=1$.

7) $x y \mapsto z=y \mapsto(x \mapsto z), x y \rightarrow z=x \rightarrow(y \rightarrow z)$.

8) $(x \vee y) \rightarrow z=(x \rightarrow z) \wedge(y \rightarrow z)$,

$(x \vee y) \mapsto z=(x \mapsto z) \wedge(y \mapsto z)$.

9) $x \rightarrow(y \wedge z)=(x \rightarrow y)(x \rightarrow z)$, $x \rightarrow(y \wedge z)=(x \rightarrow y)(x \rightarrow z)$.

If bounded integral residuated lattice $L$ is complete, then

$$
x \rightarrow z=\vee\{y \in L \mid y x \leq z\}, x \mapsto z=\vee\{y \in L \mid x y \leq z\}
$$

Thus, it follows from some results in [7] that

Theorem 1.2. Let $L$ be a complete bounded integral residuated lattice and $a, b, a_{j}, b_{j} \in L(j \in J)$. Then the following properties hold.

1) $a\left(\vee_{j \in J} b_{j}\right)=\vee_{j \in J} a b_{j}$ and $\left(\vee_{j \in J} a_{j}\right) b=\vee_{j \in J} a_{j} b$, i.e., the operation - is infinitely $\vee$-distributive.

2) $\left(\vee_{j \in J} a_{j}\right) \rightarrow b=\wedge_{j \in J}\left(a_{j} \rightarrow b\right)$ and $\left(\vee_{j \in J} a_{j}\right) \mapsto b=\wedge_{j \in J}\left(a_{j} \mapsto b\right)$.

3) $a \rightarrow\left(\wedge_{j \in J} b_{j}\right)=\wedge_{j \in J}\left(a \rightarrow b_{j}\right)$ and $a \mapsto\left(\wedge_{j \in J} b_{j}\right)=\wedge_{j \in J}\left(a \mapsto b_{j}\right)$, i.e., the two residuation operations $\rightarrow$ and $\mapsto$ are all right infinitely $\wedge$-distributive (see [8]).

4) $\left(\wedge_{j \in J} a_{j}\right) \rightarrow b \geq \vee_{j \in J}\left(a_{j} \rightarrow b\right)$ and $\left(\wedge_{j \in J} a_{j}\right) \mapsto b \geq \vee_{j \in J}\left(a_{j} \rightarrow b\right)$. 
5) $a \rightarrow\left(\vee_{j \in J} b_{j}\right) \geq \vee_{j \in J}\left(a \rightarrow b_{j}\right)$ and

$a \mapsto\left(\vee_{j \in J} b_{j}\right) \geq \vee_{j \in J}\left(a \mapsto b_{j}\right)$.

Let us define on $L$ two negations, $\neg^{L}$ and $\neg^{R}$ :

$\neg^{L} x=x \rightarrow 0$ and $\neg^{R} x=x \mapsto 0$.

For any $x, x_{j}(j \in J), b \in L$, it follows from Theorems 1.1 and 1.2 that

$$
\begin{aligned}
& \neg^{L} \neg^{R} x \geq x, \quad \neg^{R} \neg^{L} x \geq x, \quad x \rightarrow \neg^{L} y=\neg^{L}(x y), \\
& x \mapsto \neg^{R} y=\neg^{R}(x y), \quad x \rightarrow \neg^{R} y=y \mapsto \neg^{L} x, \\
& \neg^{L} \neg^{R} \neg^{L} x=\neg^{L} x, \quad \neg^{R} \neg^{L} \neg^{R} x=\neg^{R} x, \\
& x \mapsto y \leq \neg^{R} y \rightarrow \neg^{R} x, \quad x \rightarrow y \leq \neg^{L} y \mapsto \neg^{L} x, \\
& \neg^{L}\left(\vee_{j \in J} x_{j}\right)=\wedge_{j \in J} \neg^{L} x_{j}, \quad \neg^{R}\left(\vee_{j \in J} x_{j}\right)=\wedge_{j \in J} \neg^{R} x_{j}, \\
& \neg\left(\wedge_{j \in J} x_{j}\right) \geq \wedge_{j \in J} \neg^{L} x_{j}, \quad \neg^{R}\left(\wedge_{j \in J} x_{j}\right) \geq \wedge_{j \in J} \neg^{R} x_{j} .
\end{aligned}
$$

A bounded residuated lattice $L$ is called an involutive residuated lattice (see [3]) if $\neg \neg^{L} \neg^{R} X=\neg^{R} \neg^{L} X=X$ for any $x \in L$. In a complete involutive residuated lattice $L$,

$$
\begin{aligned}
& x \mapsto y=\neg^{R} y \rightarrow \neg^{R} x, x \rightarrow y=\neg^{L} y \mapsto \neg^{L} x, \\
& \neg{ }^{L}\left(\wedge_{j \in J} x_{j}\right)=\wedge_{j \in J} \neg^{L} x_{j}, \neg^{R}\left(\wedge_{j \in J} x_{j}\right)=\wedge_{j \in J} \neg^{R} x_{j} .
\end{aligned}
$$

In the sequel, unless otherwise stated, $L$ always represents any given complete bounded integral residuated lattice with maximal element 1 and minimal element 0 .

The family of all $L$-fuzzy set in $X$ will be denoted by $L^{X}$. For any family $\mu, \mu_{j} \in L^{X}(j \in J)$ of $L$-fuzzy sets, we will write $\neg^{L} \mu, \neg^{R} \mu, \vee_{j \in J} \mu_{j}$ and $\wedge_{j \in J} \mu_{j}$ to denote the $L$-fuzzy sets in $X$ given by

$$
\begin{aligned}
& \left(\neg^{L} \mu\right)(x)=\neg^{L}\left(\mu((x)),\left(\neg^{R} \mu\right)(x)=\neg^{R}(\mu((x)),\right. \\
& \left(\vee_{j \in J} \mu_{j}\right)(x)=\vee_{j \in J} \mu_{j}(x),\left(\wedge_{j \in J} \mu_{j}\right)(x)=\wedge_{j \in J} \mu_{j}(x) .
\end{aligned}
$$

Besides this, we define $1_{X}, 0_{X} \in L^{X}$ as follows: $1_{X}(x)=1 \forall x \in X$ and $0_{X}(x)=0 \forall x \in X$.

\section{L-Topological Spaces}

A completely distributive lattice $L$ is called a $F$-lattice, if $L$ has an order-reversing involution ' $L \rightarrow L$. When $L$ is a F-lattice, Liu and Luo [9] studied the concept of $L$ topology. Below, we consider the notion of $L$-topological space based on a complete bounded integral residuated lattice.

Definition 2.1. Let $\tau \subseteq L^{X}$. If $\tau$ satisfies the following three conditions:
(LFT1) $0_{X}, 1_{X} \in \tau$,
(LFT2) $\mu, v \in \tau \Rightarrow \mu \wedge v \in \tau$,
(LFT3) $\mu_{j} \in \tau \Rightarrow \vee_{j \in J} \mu_{j} \in \tau$,

then $\tau$ is called an $L$-topology on $X$ and $\left(L^{X}, \tau\right) L$ topological space.
When $L=[0,1]$, called an $L$-topological space $\left(L^{X}, \tau\right)$ an $F$-topological space.

Every element in $\tau$ is called an open subset in $L^{X}$.

Let $\tau_{L}^{\prime}=\left\{\neg^{L} \mu \mid \mu \in \tau\right\}$ and $\tau_{R}^{\prime}=\left\{\neg^{R} \mu \mid \mu \in \tau\right\}$. The elements of $\tau_{L}^{\prime}$ and $\tau_{R}^{\prime}$ are called, respectively, left closed subsets and right closed subsets in $L^{X}$.

Definition 2.2. Let $\tau$ be an $L$-topology on $X$ and $\mu$ $L$-fuzzy subset of $X$. The interior, left closure and right closure of $\mu$ w.r.t $\tau$ are, respectively, defined by

$$
\begin{aligned}
& \operatorname{int}(\mu)=\vee\{\eta \in \tau \mid \eta \leq \mu\}, \\
& c l_{L}(\mu)=\wedge\left\{\xi \in \tau_{L}^{\prime} \mid \mu \leq \xi\right\}, \\
& c l_{R}(\mu)=\wedge\left\{\zeta \in \tau_{R}^{\prime} \mid \mu \leq \zeta\right\} .
\end{aligned}
$$

int, $c l_{L}$ and $c l_{R}$ are, respectively, called interior, left closure and right closure operators.

For the sake of convenience, we denote $\operatorname{int}(\mu)$, $c l_{L}(\mu)$, and $c l_{R}(\mu)$ by $\mu^{o}, \mu_{L}^{-}$and $\mu_{R}^{-}$, respectively.

In view of Definitions 2.1 and 2.2, for any $\mu \in L^{X}$,

$$
\begin{aligned}
\mu^{o} & =\vee\{\eta \in \tau \mid \eta \leq \mu\} \in \tau, \\
\mu_{L}^{-} & =\wedge\left\{\neg^{L} \xi \mid \mu \leq \neg^{L} \xi, \xi \in \tau\right\} \\
& =\neg^{L}\left(\vee\left\{\xi \mid \mu \leq \neg^{L} \xi, \xi \in \tau\right\}\right)=\neg^{L} \mu_{1}, \\
\mu_{R}^{-} & =\wedge\left\{\neg^{R} \zeta \mid \mu \leq \neg^{R} \zeta, \zeta \in \tau\right\} \\
& =\neg^{R}\left(\vee\left\{\zeta \mid \mu \leq \neg^{R} \zeta, \zeta \in \tau\right\}\right)=\neg^{R} \mu_{2},
\end{aligned}
$$

where

$$
\begin{aligned}
& \mu_{1}=\vee\left\{\xi \mid \mu \leq \neg^{L} \xi, \xi \in \tau\right\} \in \tau, \\
& \mu_{2}=\vee\left\{\zeta \mid \mu \leq \neg^{R} \zeta, \zeta \in \tau\right\} \in \tau,
\end{aligned}
$$

i.e., $\mu^{o}$ is just the largest open subset contained in $\mu$, $\mu_{L}^{-}$and $\mu_{L}^{-}$are, respectively, the smallest left closed and right closed subsets containing $\mu$.

For any $\mu \in L^{X}$,

$$
\begin{aligned}
\neg^{L}\left(\mu^{o}\right) & =\neg^{L}(\vee\{\eta \in \tau \mid \eta \leq \mu\})=\wedge\left\{\neg^{L} \eta \mid \eta \leq \mu, \eta \in \tau\right\} \\
& \geq \wedge\left\{\neg^{L} \eta \mid \neg^{L} \mu \leq \neg^{L} \xi, \eta \in \tau\right\}=\left(\neg^{L} \mu\right)_{L}^{-} .
\end{aligned}
$$

Similarly, $\neg^{R}\left(\mu^{o}\right) \geq\left(\neg^{R} \mu\right)_{R}^{-}$.

Theorem 2.1. If $L$ is an involutive residuated lattice and $\mu \in L^{X}$, then
1) $\neg^{L}\left(\mu^{o}\right)=\left(\neg^{L} \mu\right)_{L}^{-}$and $\neg^{R}\left(\mu^{o}\right)=\left(\neg^{R} \mu\right)_{R}^{-}$;
2) $\mu^{o}=\neg^{L}\left(\neg^{R} \mu\right)_{R}^{-}=\neg^{R}\left(\neg^{L} \mu\right)_{L}^{-}$;
3) $\left(\neg^{L} \mu\right)^{o}=\neg^{L} \mu_{R}^{-},\left(\neg^{R} \mu\right)^{o}=\neg^{R} \mu_{L}^{-}$, 
$\mu_{L}^{-}=\neg^{L}\left(\neg^{R} \mu\right)^{o}$ and $\mu_{R}^{-}=\neg^{R}\left(\neg^{L} \mu\right)^{o}$.

Proof. When $L$ is an involutive residuated lattice, $\neg^{R}\left(\neg^{L} \mu\right)=\neg^{L}\left(\neg^{R} \mu\right)=\mu \forall \mu \in L^{X}$.

1) If $\eta \in L^{X}$ and $\neg^{L} \mu \leq \neg^{L} \eta$, then

$$
\mu=\neg^{R}\left(\neg^{L} \mu\right) \geq \neg^{R}\left(\neg^{L} \eta\right)=\eta .
$$

Thus, $\neg^{L}\left(\mu^{o}\right)=\left(\neg^{L} \mu\right)_{L}^{-}$. Similarly,

$\neg^{R}\left(\mu^{o}\right)=\left(\neg^{R} \mu\right)_{R}^{-}$.

2) It follows from 1) that

$$
\begin{aligned}
& \mu^{o}=\neg^{R} \neg^{L}\left(\mu^{o}\right)=\neg^{R}\left(\neg^{L} \mu\right)_{L}^{-}, \\
& \mu^{o}=\neg^{L} \neg^{R}\left(\mu^{o}\right)=\neg^{L}\left(\neg^{R} \mu\right)_{R}^{-} .
\end{aligned}
$$

3) By 2), we see that

$$
\begin{gathered}
\left(\neg^{L} \mu\right)^{o}=\neg^{L}\left(\neg^{R} \neg^{L} \mu\right)_{R}^{-}=\neg^{L}\left(\mu_{R}^{-}\right), \\
\left(\neg^{R} \mu\right)^{o}=\neg^{R}\left(\neg^{L} \neg^{R} \mu\right)_{L}^{-}=\neg^{R}\left(\mu_{L}^{-}\right), \\
\neg^{L}\left(\neg^{R} \mu\right)^{o}=\neg^{L}\left(\neg^{R}\left(\neg^{L} \neg^{R} \mu\right)_{L}^{-}\right)=\mu_{L}^{-}, \\
\neg^{R}\left(\neg^{L} \mu\right)^{o}=\neg^{R}\left(\neg^{L}\left(\neg^{R} \neg^{L} \mu\right)_{R}^{-}\right)=\mu_{R}^{-} .
\end{gathered}
$$

Theorem 2.2. Let $\mu, v \in L^{X}$. Then the following properties hold:

1) $\left(1_{X}\right)^{o}=1_{X},\left(0_{X}\right)_{L}^{-}=\left(0_{X}\right)_{R}^{-}=0_{X}$.

2) $\mu^{o} \leq \mu, \mu \leq \mu_{L}^{-}, \mu \leq \mu_{R}^{-}$.

3) If $\mu \leq v$, then $\mu^{o} \leq v^{o}, \mu_{L}^{-} \leq v_{L}^{-}, \mu_{R}^{-} \leq v_{R}^{-}$.

4) $\left(\mu^{o}\right)^{o}=\mu^{o},\left(\mu_{L}^{-}\right)_{L}^{-}=\mu_{L}^{-}$and $\left(\mu_{R}^{-}\right)_{R}^{-}=\mu_{R}^{-}$.

5) $(\mu \wedge v)^{o}=\mu^{o} \wedge v^{o}$.

6) If $\neg^{L}(x \wedge y)=\neg^{L} x \vee \neg^{L} y \forall x, y \in L$, then

$(\mu \vee v)_{L}^{-}=\mu_{L}^{-} \vee v_{L}^{-}$.

7) If $\neg^{R}(x \wedge y)=\neg^{R} x \vee \neg^{R} y \forall x, y \in L$, then $(\mu \vee v)_{R}^{-}=\mu_{R}^{-} \vee v_{R}^{-}$.

Proof. By Definition 2.2, it is easy to see that 1)-3) hold.

4) By 2) and 3), we have that $\left(\mu^{o}\right)^{o} \leq \mu^{\circ}$. On the other hand, $\mu^{o} \in \tau$ and $\mu^{o} \leq \mu^{o}$. Thus, it follows from Definition 2.1 that $\mu^{o} \leq\left(\mu^{o}\right)^{o}$ and so $\left(\mu^{o}\right)^{o}=\mu^{o}$. We can prove in an analogous way that $\left(\mu_{L}^{-}\right)_{L}^{-}=\mu_{L}^{-}$and $\left(\mu_{R}^{-}\right)_{R}^{-}=\mu_{R}^{-}$.
5) Clearly, $(\mu \wedge v)^{o} \leq \mu^{o} \wedge v^{o}$. Noting that $\mu^{o} \wedge v^{o} \in \tau$, we see that

$$
\mu^{o} \wedge v^{o}=\left(\mu^{o} \wedge v^{o}\right)^{o} \leq \mu^{o} \wedge v^{o} .
$$

Thus, $(\mu \wedge v)^{o}=\mu^{o} \wedge v^{o}$.

6) There exist $\mu_{1}, v_{1} \in \tau$ such that $\mu_{L}^{-}=\neg^{L} \mu_{1}$, $v_{L}^{-}=\neg^{L} v_{1}$.

If $\neg^{L}(x \wedge y)=\neg^{L} x \vee \neg^{L} y \forall x, y \in L$, then $\mu \vee v \leq \mu_{L}^{-} \vee v_{L}^{-}=\neg^{L} \mu_{1} \vee \neg^{L} v_{1}=\neg^{L}\left(\mu_{1} \wedge v_{1}\right) \in \tau_{L}^{-}$. Thus, $(\mu \vee v)_{L}^{-} \leq \mu_{L}^{-} \vee v_{L}^{-}$. Clearly, $(\mu \vee v)_{L}^{-} \geq \mu_{L}^{-} \vee v_{L}^{-}$.

Therefore, $(\mu \vee v)_{L}^{-}=\mu_{L}^{-} \vee v_{L}^{-}$.

7) Similar to (6).

Theorem 2.3. Let $f: L^{X} \rightarrow L^{X}$ be a mapping. Then the following two statements hold.

1) If the operator $f$ on $L^{X}$ satisfying the follwing conditions:

(C1) $f\left(1_{X}\right)=1_{X}$,

(C2) $f(\mu) \leq \mu \forall \mu \in L^{X}$,

(C3) $f(\mu \wedge v)=f(\mu) \wedge f(v) \forall \mu, v \in L^{X}$, then $\tau=\left\{\xi \mid f(\xi)=\xi, \xi \in L^{X}\right\}$ is an L-topology on $X$. Moreover, if the operator falso fulfills

(C4) $f(f(\mu))=f(\mu) \forall \mu \in L^{X}$, then with the L-topology $\tau, f(\mu)=\mu^{o}$ for every $\mu \in L^{X}$, i.e., $f$ is the interior operator w.r.t $\tau$.

2) If the operator $f$ on $L^{X}$ satisfying the follwing conditions:

(C1') $f\left(0_{X}\right)=0_{X}$,

(C2') $\mu \leq f(\mu) \forall \mu \in L^{X}$,

(C3') $f(\mu \vee v)=f(\mu) \vee f(v) \forall \mu, v \in L^{X}$, then

a) when $\neg^{L}(x \wedge y)=\neg^{L} x \vee \neg^{L} y \forall x, y \in L$,

$$
\tau_{1}=\left\{\eta \mid f\left(\neg^{L} \eta\right)=\neg^{L} \eta, \eta \in L^{X}\right\}
$$

is an L-topology on $X$, moreover, if the operator $f$ also fulfills

(C4) $f(f(\mu))=f(\mu) \forall \mu \in L^{X}$, and $\neg^{L}: L^{X} \rightarrow L^{X}$ is a bijection, then with the L-topology $\tau_{1}$,

$f(\mu)=\mu_{L}^{-} \forall \mu \in L^{X}$, i.e., $f$ is the left closure operator w.r.t $\tau_{1}$

b) when $\neg^{R}(x \wedge y)=\neg^{R} x \vee \neg^{R} y \forall x, y \in L$,

$$
\tau_{2}=\left\{\xi \mid f\left(\neg^{R} \xi\right)=\neg^{R} \xi, \xi \in L^{X}\right\}
$$

is also an L-topology on X, moreover if (C4) holds and $\neg^{R}: L^{X} \rightarrow L^{X}$ is a bijection, then with the L-topology $\tau_{2}, f(\mu)=\mu_{R}^{-} \forall \mu \in L^{X}$, i.e., $f$ is the right closure operator w.r.t $\tau_{2}$.

Proof. 1) Refer to the proof of Theorem 2.2.21 in [9].

2) Clearly, $0_{X}, 1_{X} \in \tau_{1}$. If $\eta_{1}, \eta_{2} \in \tau_{1}$, then

$$
\begin{aligned}
f\left(\neg^{L}\left(\eta_{1} \wedge \eta_{2}\right)\right) & =f\left(\neg \neg^{L} \eta_{1} \vee \neg^{L} \eta_{2}\right) \\
& =f\left(\neg^{L} \eta_{1}\right) \vee f\left(\neg^{L} \eta_{2}\right)=\neg^{L} \eta_{1} \vee \neg^{L} \eta_{2} \\
& =\neg^{L}\left(\eta_{1} \wedge \eta_{2}\right),
\end{aligned}
$$

i.e., $\eta_{1} \wedge \eta_{2} \in \tau_{1}$. If $\eta_{j} \in \tau_{1}(j \in J)$, then 


$$
\begin{aligned}
f\left(\neg^{L}\left(\vee_{j \in J} \eta_{j}\right)\right) & =f\left(\wedge_{j \in J} \neg{ }^{L} \eta_{j}\right) \leq \wedge_{j \in J} f\left(\neg \neg^{L} \eta_{j}\right) \\
& =\wedge_{j \in J} \neg^{L} \eta_{j}=\neg^{L}\left(\vee_{j \in J} \eta_{j}\right) .
\end{aligned}
$$

Combing with (C2'), we have that

$$
f\left(\neg^{L}\left(\vee_{j \in J} \eta_{j}\right)\right)=\neg^{L}\left(\vee_{j \in J} \eta_{j}\right) .
$$

Thus, $\vee_{j \in J} \eta_{j} \in \tau_{1}$ and so $\tau_{1}$ is an $L$-topology on $X$. For any $\mu \in L^{X}$,

$$
\begin{aligned}
f\left(\mu_{L}^{-}\right) & =f\left(\wedge\left\{\neg^{L} \xi \mid \mu \leq \neg^{L} \xi, \xi \in \tau_{1}\right\}\right) \\
& \leq\left(\wedge\left\{f\left(\neg^{L} \xi\right) \mid \mu \leq \neg^{L} \xi, \xi \in \tau_{1}\right\}\right) \\
& \left.=\wedge\left\{\neg^{L} \xi \mid \mu \leq \neg^{L} \xi, \xi \in \tau_{1}\right\}\right)=\mu_{L}^{-},
\end{aligned}
$$

i.e., $f(\mu) \leq f\left(\mu_{L}^{-}\right) \leq \mu_{L}^{-}$. Moreover, if (C4) holds and $\neg^{L}: L^{X} \rightarrow L^{X}$ is a bijection, then

$$
\begin{aligned}
f(\mu) & \geq \wedge\left\{\eta \in L^{X} \mid f(\eta)=\eta \geq \mu\right\} \\
& =\wedge\left\{\neg^{L} \xi \mid \mu \leq \neg^{L} \xi, \xi \in \tau_{1}\right\}=\mu_{L}^{-} .
\end{aligned}
$$

Therefore, $f(\mu)=\mu_{L}^{-}$, i.e., $f$ is the left closure operator w.r.t $\tau_{1}$.

We can prove in an analogous way that $\tau_{2}$ is an $L$ topology on $X$ and the corresponding $f$ is the right closure operator w.r.t $\tau_{2}$.

\section{Acknowledgements}

This work is supported by Science Foundation of Yancheng Teachers University (11YSYJB0201).

\section{REFERENCES}

[1] K. Blount and C. Tsinakis, "The Structure of Residuated Lattices,” International Journal of Algebra and Computation, Vol. 13, No. 4, 2003, pp. 437-461.

[2] N. Galatos, P. Jipsen, T. Kowalski and H. One, "Residuated Lattices: An Algebraic Glimpse at Substructural Logics,” Elsevier, Amsterdam, 2007.

[3] L. Z. Liu and K. T. Li, "Boolean Filters and Positive Implicative Filters of Residuated Lattices,” Information Sciences, Vol. 177, No. 24, 2007, pp. 5725-5738. doi:10.1016/j.ins.2007.07.014

[4] Z. D. Wang and J. X. Fang, “On v-Filters and Normal v-Filters of a Residuated Lattice with a Weak vt-Operator," Information Sciences, Vol. 178, No. 17, 2008, pp. 3465-3473. doi:10.1016/j.ins.2008.04.003

[5] U. Hohle, "Commutative, Residuated L-Monoids," In: U. Hohle and E. P. Klement, Eds., Non-Classical Logics and Their Applications to Fuzzy Subsets, Kluwer Academic Publishers, Boston, Dordrecht, 1995, pp. 53-106.

[6] A. M. Radzikowska and E. E. Kerre, "Fuzzy Rough Sets Based on Residuated Lattices,” In: J. F. Peter et al., Eds., Transactions on Rough Sets II, LNCS 3135, 2004, pp. 278-296.

[7] Z. D. Wang and Y. D. Yu, "Pseudo-t-Norms and Implication Operators on a Complete Brouwerian Lattice,” Fuzzy Sets and Systems, Vol. 132, No. 1, 2002, pp. 113-124. doi:10.1016/S0165-0114(01)00210-X

[8] Z. D. Wang and J. X. Fang, "Residual Operations of Left and Right Uninorms on a Complete Lattice,” Fuzzy Sets and Systems, Vol. 160, No. 1, 2009, pp. 22-31. doi:10.1016/j.fss.2008.03.001

[9] Y. M. Liu and M. K. Luo, "Fuzzy Topology,” World Scientific Publishing, Singapore, 1997. 